https://doi.org/10.29296/25877305-2018-12-02

\section{Повышение комплаентности}

\section{при эрадикационной терапии}

\section{хронического Нр-ассоциированного}

\section{гастрита}

Е. Авалуева, доктор медицинских наук, профессор,

Е. Сказываева, кандидат медицинских наук,

И. Бакулин, доктор медицинских наук, профессор,

с. Ситкин, кандидат медицинских наук

Северо-Западный государственный медицинский университет

им. И.И. Мечникова, Санкт-Петербург

E-mail: sitkins@mail.ru

Дополнительное назначение препарата масляной кислоты (Закофальк NMX) при эрадикации Helicobacter pylori у пациентов с хроническим гастритом способствует уменьшению диарейного синдрома и улучшению качества жизни, что повышает комплаентность пациентов.

Ключевые слова: гастроэнтерология, Helicobacter pylori, эрадикационная терапия, хронический гастрит, комплаентность.

Для цитирования: Авалуева Е., Сказываева Е., Бакулин И. др. Повышение комплаентности при эрадикационной терапии хронического Нpассоциированного гастрита // Врач. - 2018; 29 (12): 9-14. https://doi. org/10.29296/25877305-2018-12-02

$\mathrm{C}$ конца 80-х годов XX века стандартная тройная терапия, основанная на использовании ингибитора протонной помпы (ИПП), антибактериальных препаратов - АБП (кларитромицин, амоксициллин или метронидазол) была «золотым стандартом» в лечении инфекции Helicobacter pylori (Hp). Отмечаемое в настоящее время снижение эффективности эрадикационной терапии (ЭТ) $[1,2]$ связывают с факторами, ассоциированными с $H p$, и ухудшением комплаентности пациентов.

Среди факторов, ассоциированных с $H p$, отмечают учащение резистентности $Н p$ к АБП, особенно к кларитромицину, что снижает эффективность стандартных режимов лечения, увеличивает длительность лечения АБП и требует использования в схемах терапии большего их количества [1-6].

Одним из важнейших факторов неэффективности ЭТ является снижение приверженности лечению (комплаентности пациента). У больных с хорошим комплаенсом отмечается более высокая, чем при низком комплаенсе, эффективность ЭТ (соответственно 96 и 69\%) [3, 7]. Пациентам с гастритом свойствен высокий уровень общей комплаентности; больные стремятся вступать в доверительные отношения с врачом, опираться на его мнение, они озабочены впечатлением, производимым на окружающих, в частности на врача, который воспринимается ими как значимое лицо [7]. Как показали данные последнего метаанализа, снижение приверженности лечению пациентов с Hp-поражением пищеварительного тракта не зависит от количества принимаемых одновременно таблеток, а связано с кратностью приема препаратов в течение суток и продолжительностью курса лечения, который, согласно последним рекомендациям, составляет 14 дней [8]. Увеличение длительности ЭТ, прием одновременно двух АБП, с одной стороны, способствует повышению эффективности эрадикации, а с другой - чаще приводит к нежелательным эффектам терапии, в частности, связанным с развитием дисбиотических процессов в пищеварительном тракте. Так, у $28-48 \%$ пациентов отмечаются явления желудочной и кишечной диспепсии, связанные с нарушением микробиоты желудочно-кишечного тракта (ЖКТ) [9]. Развитие нежелательных явлений, возникающих на фоне ЭТ, - одна из основных причин снижения комплаенса у пациентов с $Н p$-ассоциированным гастритом [3, 9].

С появлением молекулярно-генетических методов исследования микробиоты появилась возможность по-новому оценить нарушения микрофлоры на фоне антибактериальной терапии, в частности, при эрадикации Нр. Так, показано, что при приеме АБП в первую очередь уменьшается количество бактерий, образующих масляную кислоту (бутират-продуцентов), а не бифидо- и лактобактерий, как считалось ранее [10]. При $H p$-позитивном гастрите изначально существует дефицит бактерий, синтезирующих масляную кислоту [11]. На фоне ЭТ количество бутиратпродуцирующих бактерий существенно уменьшается уже на 2-4-е сутки лечения [12].

Нарушение метаболической активности микрофлоры приводит к дефициту ключевого ее метаболита масляной кислоты (бутирата). Масляная кислота является основным регулятором водно-электролитного баланса в толстой кишке, выступает в качестве энергетического и регулирующего субстрата для колоноцитов, способствуя развитию и обновлению клеток слизистой оболочки толстой кишки (рис. 1). Возникающий на фоне ЭТ дефицит масляной кислоты приводит к нарушению бутират-стимулированной абсорбции воды и электролитов, снижению энергообеспечения и диспролиферации кишечного эпителия (рис. 2). Клиническим проявлением данных нарушений у большинства (80-90\%) пациентов служит идиопатическая ААД, этиологически не связанная с каким-либо инфекционным агентом и характеризующаяся следующими симптомами: вздутие живота с избыточным газовыделением, абдоминальный дискомфорт, послабление стула без патологических примесей в кале (без крови и слизи). 
В связи со сказанным можно предположить, что дополнительное назначение на фоне ЭТ препаратов, восполняющих дефицит масляной кислоты, позволит снизить количество побочных эффектов указанной терапии, ассоциированных с изменением микробиоты пищеварительного тракта, опосредованно повысит комплаенс пациента и в итоге - эффективность эрадикации $[1,3,9]$.

Зарегистрированный в России препарат Закофальк NMX является биологически активной добавкой к пище; он обладает уникальным составом и соединяет масляную кислоту и пребиотик инулин, заключенные в таблетку с полимерной мультиматриксной структурой с высвобождением действующих веществ непосредственно в толстой кишке на всем ее протяжении.

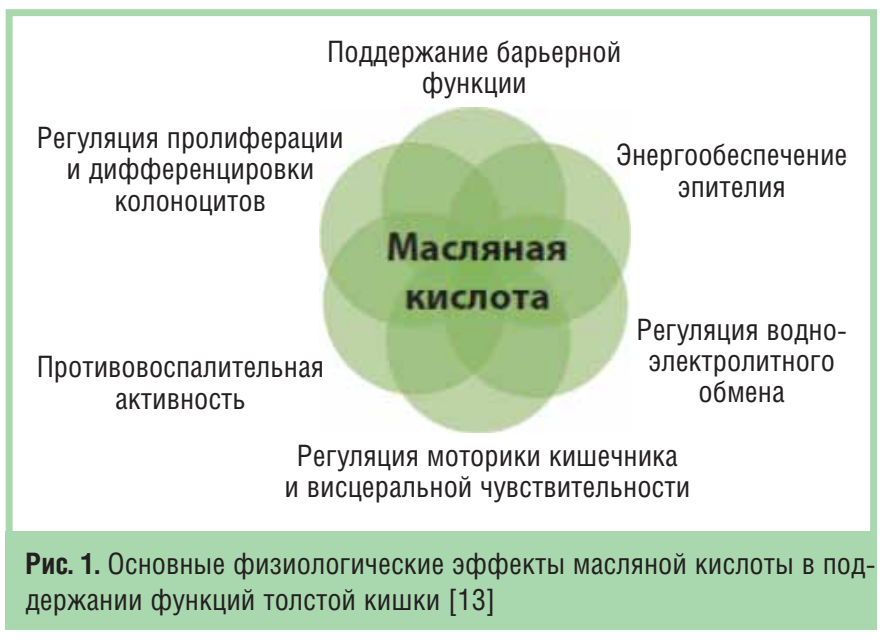

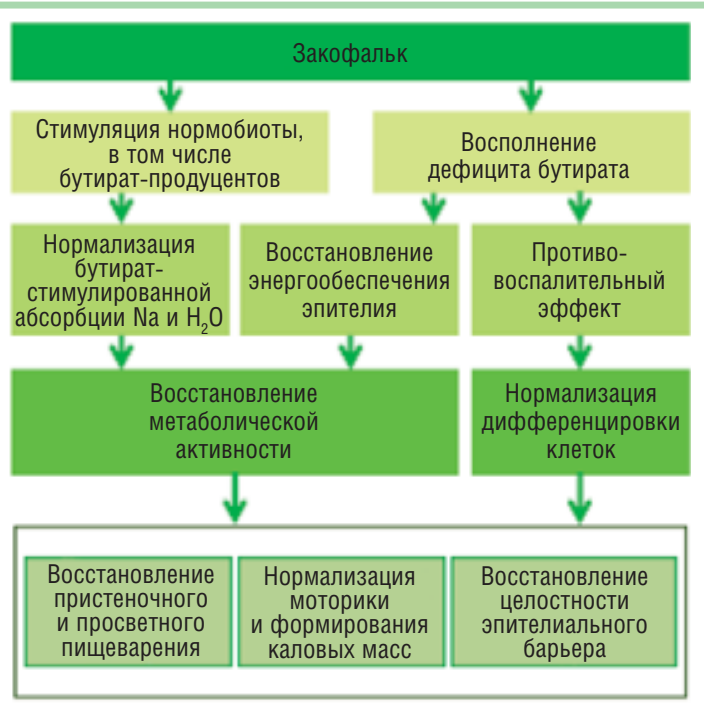

Предотвращение/минимизация/ликвидация симптомов ААД

Рис. 2. Роль десицита бутирата в развитии идиопатической ААД. Анти-ААД-эффекты Закофралька [14]. Примечание. ААД - антибиотикоассоциированная диарея

Целью нашего исследования было определение эффективности препарата Закофальк NMX, назначаемого для повышения приверженности лечению и уменьшения нежелательных явлений у пациентов с $H p$-ассоциированными заболеваниями верхних отделов пищеварительного тракта, возникающих в процессе ЭТ.

\section{МАТЕРИАЛ И МЕТОДЫ}

В исследовании участвовали 50 амбулаторных пациентов с $H p$-ассоциированным хроническим гастритом. Инфицирование $H p$ подтверждалось уреазным тестом, выполненным во время фиброэзофагогастродуоденоскопии (ФГДС); для выявления микроорганизмов использовали быстрый уреазный тест, диски индикаторная бумага. Согласно данным теста, Hp были выявлены у 100\% включенных в исследование пациентов. Диагноз хронического гастрита у всех пациентов был установлен при ФГДС и подтвержден гистологически. Морфологическое исследование гастробиоптатов проводили в соответствии с гистотехнологическим стандартом. Хронический гастрит до лечения был документирован у всех 50 обследованных, в том числе у 29 (58\%) пациентов с умеренной и резко выраженной инфильтрацией клетками мононуклеарного ряда.

Возраст больных варьировал от 20 до 48 лет (средний возраст - 33,2 4,4 года); мужчин было 23 , женщин -27 . Достоверных различий между группами по возрасту и сопутствующим заболеваниям не было. В исследование не включали больных с указанием в анамнезе на осложнения болезней верхних отделов ЖКТ и пациентов с тяжелой сопутствующей патологией.

Обследованные были разделены на 2 группы по 25 человек. Пациенты 1-й группы получали ЭТ 1-й линии (ИПП в стандартной дозе, амоксициллин 1000 мг 2 раза в день, кларитромицин - 500 мг 2 раза в день в течение 14 дней) и дополнительно препарат Закофальк NMX (по 1 капсуле 2 раза в день); пациенты 2-й группы - только 14-дневную ЭТ.

Пациенты обеих групп до начала лечения, а также через 7 и 14 дней после начала ЭТ заполняли стандартизированный опросник жалоб пациента, опросник качества жизни (КЖ) гастроэнтерологических больных GSRS, опросник 
KЖ SF-36. Интенсивность признака в стандартизированном опроснике оценивали по условной шкале в баллах: 0 баллов - нет признака, 1 балл - признак присутствует.

Статистическая обработка полученных результатов проводилась по программам, включенным в пакет MS Excel, с использованием библиотеки статистических функций. Для оценки достоверности данных использовали непараметрический критерий Пирсона $\chi^{2}$. Показатели выражали в виде $\mathrm{M} \pm \mathrm{s}$, где $\mathrm{M}$ - среднее значение, $\mathrm{s}-$ среднее квадратичное отклонение. Достоверными считали различия при $\mathrm{p}<0,05$.

\section{РЕЗУЛЬТАТЫ И ОБСУЖДЕНИЕ}

При опросе пациентов до начала лечения достоверных различий между группами не выявлено. Большинство обследованных жаловались на общую слабость, боли в животе (чаще натощак, с локализацией в эпигастральной области, реже - диффузные), абдоминальный дискомфорт, вздутие живота, тошноту, нарушение регулярности стула.

После лечения у всех пациентов 1-й группы отмечены положительная динамика самочувствия, уменьшение признаков желудочной и кишечной диспепсии. Во 2-й группе через 14 дней наблюдения самочувствие улучшилось у 11 (44\%) пациентов; однако на фоне улучшения самочувствия, купирования или уменьшения выраженности абдоминального болевого синдрома отмечалось усиление симптомов кишечной диспепсии (вздутие и урчание в животе, частое отхождение газов, склонность к послаблению стула). Результаты стандартизированного опроса показали достоверное по сравнению с 1-й группой увеличение к 14-му дню лечения выраженности симптомов кишечной диспепсии (табл. 1).

Таким образом, включение препарата Закофальк в схему ЭТ улучшает переносимость лечения АБП. Восполняя дефицит бутирата (как непосредственно за счет готового метаболита, так и вследствие стимуляции нормальной бутират-стимулирующей микробиоты), Закофальк нормализует абсорбцию натрия и воды из просвета кишки, восстанавливает энергоснабжение эпителия, оказывает противовоспалительный эффект, тем самым способствуя восстановлению процессов пищеварения, моторики и защитных функций эпителиального барьера. Это в итоге приводит к предотвращению или ускоренному регрессу симптомов идиопатической ААД (см. рис. 2).

Результаты оценки приверженности пациентов терапии показаны в табл. 2: она составила при приеме ИПП - в обеих группах 99\%, при приеме препарата Закофальк NMX (1-я группа) - 99\%, при приеме АБП: в 1-й группе - 96\%, во 2-й - 92\% (p=0,009). Кроме того, во 2-й группе у $3(12 \%)$ пациентов отмечались учащение стула до 3 раз в сутки на 9-10-й день терапии и изменение его характера (5-6-й тип

\section{ИННОВАЦИОННЫЙ ПОДХОД К ЗДОРОВЬЮ КИШЕЧНИКА}

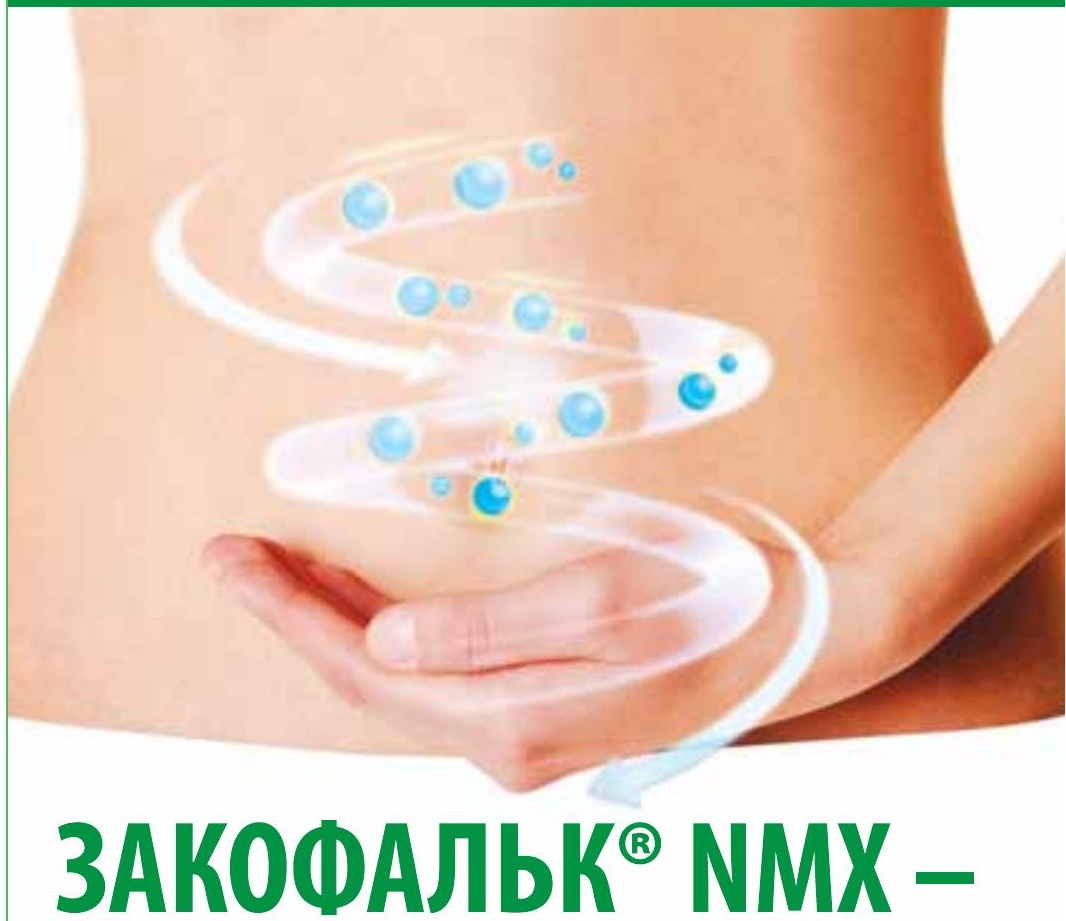

комбинированный препарат масляной кислоты и инулина

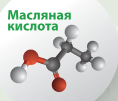

Масляная кислота - основной метаболит микрофлоры, источник энергии колоноцитов и регулятор метаболических и сигнальных процессов в кишечнике.

Инулин - природное пищевое волокно, стимулирует рост собственной микрофлоры, источник эндогенной масляной кислоты.

\section{Полимерная мультиматриксная систе-} ма NMX - инновационная лекарственная форма доставляет действующие вещества в толстую кишку с высвобождением на всем ее протяжении.
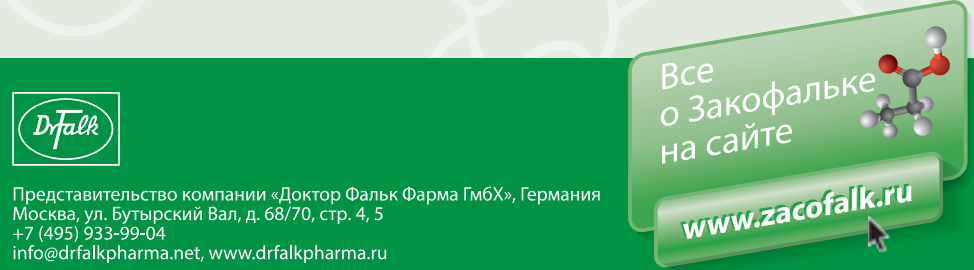


\begin{tabular}{|c|c|c|c|c|c|c|c|}
\hline \multicolumn{8}{|c|}{ Динамика субъективных проявлений у пациентов с Нр-ассоциированным гастритом; n (\%) } \\
\hline \multirow{2}{*}{ Симптомы } & \multicolumn{3}{|c|}{ 1-я группа } & \multicolumn{3}{|c|}{ 2-я группа } & \multirow{2}{*}{$\mathbf{p}_{2}$} \\
\hline & до лечения & через 14 дней & $\mathbf{p}_{1}$ & до лечения & через 14 дней & $p_{1}$ & \\
\hline Общая слабость & $13(52)$ & $7(28)$ & 0,148 & $15(60)$ & $10(40)$ & 0,257 & 0,551 \\
\hline Недомогание & $12(48)$ & $7(28)$ & 0,244 & $12(48)$ & $12(48)$ & 1,0 & 0,243 \\
\hline Абдоминальный дискомфорт & $13(52)$ & $1(4)$ & $<0,001$ & $14(56)$ & $9(36)$ & 0,256 & 0,01 \\
\hline Боли в животе & $14(56)$ & $2(8)$ & $<0,001$ & $13(52)$ & $2(8)$ & $<0,001$ & - \\
\hline Тошнота & $7(28)$ & $3(12)$ & 0,289 & $7(28)$ & $4(16)$ & 0,496 & $>0,99$ \\
\hline Отрыжка кислым & $8(32)$ & $2(20)$ & 0,07 & $5(20)$ & $0(0)$ & 0,05 & 0,489 \\
\hline Склонность к послаблению стула & $7(28)$ & $0(0)$ & 0,009 & $6(24)$ & $13(52)$ & 0,08 & $<0,001$ \\
\hline Склонность к запору & $10(40)$ & $1(4)$ & 0,004 & $12(48)$ & $4(20)$ & 0,032 & 0,348 \\
\hline
\end{tabular}

Таблица 2

Приверженность приему лекарственных препаратов в 2 группах обследованных

\begin{tabular}{|lccc|}
\hline \multirow{2}{*}{ Препарат } & \multicolumn{2}{c}{ Комплаенс, \% } & \multirow{2}{*}{ p } \\
\cline { 2 - 3 } & 1-я группа & 2-я группа & \\
\hline Амоксициллин (1000 мг 2 раза в день) & 95,7 & 92,0 & 0,106 \\
\hline Кларитромицин (500 мг 2 раза в день) & 96,4 & 91,9 & 0,043 \\
\hline АБП в целом & 96,0 & 92,0 & 0,009 \\
\hline ИПП & 99,4 & 99,1 & 0,740 \\
\hline Закосральк NMX & $99,0 \%$ & - & - \\
\hline
\end{tabular}

\begin{tabular}{|c|c|c|c|c|c|}
\hline \multicolumn{5}{|c|}{$\begin{array}{c}\text { Динамика выраженности жалоб у обследованных } 2 \text { групп } \\
\text { по данным опросника GSRS }\end{array}$} & \multirow{3}{*}{$\begin{array}{c}\text { Таблица } 3 \\
\text { p }\end{array}$} \\
\hline \multirow{2}{*}{ Шкала } & \multicolumn{2}{|c|}{ 1-я группа } & \multicolumn{2}{|c|}{ 2-я группа } & \\
\hline & $M \pm s$ & Me & $M \pm s$ & Me & \\
\hline Абдоминальная боль & $\frac{5,1 \pm 1,8}{3,9 \pm 1,3}$ & $\frac{5,0}{4,0}$ & $\frac{5,0 \pm 2,1}{4,8 \pm 1,6}$ & $\frac{5,0}{5,0}$ & 0,738 \\
\hline Рефрлюкс-синдром & $\frac{6,3 \pm 3,6}{5,1 \pm 1,6}$ & $\frac{5,0}{6,0}$ & $\frac{6,9 \pm 3,5}{5,1 \pm 1,2}$ & $\frac{6,0}{6,0}$ & 0,747 \\
\hline Диарейный синдром & $\frac{4,9 \pm 2,4}{3,9 \pm 2,1}$ & $\frac{4,0}{3,0}$ & $\frac{3,7 \pm 1,3}{7,5 \pm 2,4}$ & $\frac{3,0}{7,0}$ & $0,001^{*}$ \\
\hline $\begin{array}{l}\text { Диспепсический } \\
\text { синдром }\end{array}$ & $\frac{11,1 \pm 4,8}{8,3 \pm 3,1}$ & $\frac{12,0}{7,0}$ & $\frac{10,8 \pm 5,3}{9,7 \pm 3,3}$ & $\frac{11,0}{10,0}$ & 0,369 \\
\hline Синдром запора & $\frac{7,5 \pm 3,7}{4,1 \pm 1,2}$ & $\frac{8,0}{4,0}$ & $\frac{7,0 \pm 4,2}{3,6 \pm 0,9}$ & $\frac{5,0}{3,0}$ & $0,001^{*}$ \\
\hline $\begin{array}{l}\text { Шкала суммарного } \\
\text { измерения }\end{array}$ & $\frac{34,3 \pm 9,9}{25,6 \pm 5,4}$ & $\frac{32,0}{26,0}$ & $\frac{33,5 \pm 11,5}{30,6 \pm 6,2}$ & $\frac{30,0}{32,0}$ & 0,503 \\
\hline $\begin{array}{l}\text { Примечание. В числи } \\
\text { группами после лече }\end{array}$ & $\begin{array}{l}10 \text { лечени } \\
p<0,01\end{array}$ & & после н & разли & между \\
\hline
\end{tabular}

по Бристольской шкале), в связи с чем пациенты прекратили принимать АБП, в результате чего эрадикация Нр у них не была достигнута.

Следует отметить высокую приверженность ЭТ в обеих группах, что объясняется правильной мотивацией пациентов, включенных в исследование (ведение ими дневника приема лекарственных препаратов и контроль врачом исполнения назначений). В реальной практике приверженность ЭТ, по данным исследований, составляет 24$60 \%$ [15, 16], поскольку побочные эффекты со стороны ЖКТ вынуждают почти каждого 4-го пациента досрочно прекращать курс ЭТ, что, несомненно, снижает результаты эрадикации [17, 18]. Включение Закофалька в схему ЭТ улучшает переносимость лечения и позволяет пациенту не отказываться от приема АБП в течение всего курса, что повышает эффективность эрадикации на $20 \%$ [19].

После проведенного лечения КЖ пациентов, по данным опросника GSRS, согласно шкале суммарного измерения, достоверно улучшилось (при сравнении показателей до и после лечения в 1-й группе $p=0,035$; во 2-й $-p=0,03)$; достоверных различий между группами по окончании лечения не получено. В табл. 3 представлена динамика гастроинтестинальных жалоб по опроснику GSRS у обследованных 2 групп до и после лечения. Выявлены 
статистически значимые различия в группах по шкалам диареи и запора. После курса ЭТ жалобы на послабление стула были более выраженными у пациентов 2-й группы $(\mathrm{p}<0,01)$ : диарейный синдром в этой группе составил до лечения $3,7 \pm 1,3$ балла, после лечения $-7,5 \pm 2,4$ балла (в 1-й группе - соответственно

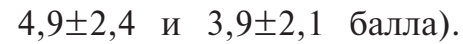
Кроме того, во 2-й группе отмечено достоверно более выраженное уменьшение синдрома запора $(\mathrm{p}<0,01)$ : показатели по шкале запора в 1-й группе до лечения составили 7,5 $\pm 3,7$ балла,

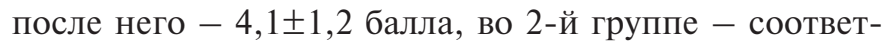

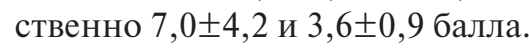

При оценке КЖ с помощью опросника SF-36 у всех обследованных в начале наблюдения отмечались высокие и средние показатели по всем шкалам, кроме ролевого функционирования, обусловленного эмоциональным состоянием (RE). После лечения в 1-й группе отмечено увеличение показателей КЖ по всем шкалам, наиболее выраженное - по шкалам GH

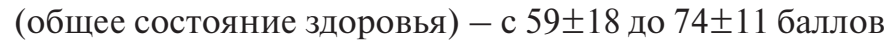
$(\mathrm{p}=0,019), \mathrm{RP}$ (ролевое функционирование) - c 64士28 до $75 \pm 18$ баллов $(\mathrm{p}=0,034)$ и МН (психическое здоровье) - с 53 11 до $72 \pm 17$ баллов $(\mathrm{p}=0,037)$. Достоверных изменений показателей КЖ во 2-й группе после лечения не было - отмечена лишь тенденция к улучшению показателей КЖ по всем шкалам (рис. 3, $a$, б).

В современной клинической практике в ходе ЭТ у пациентов с хроническим $Н p$-ассоциированным гастритом часто возникают нежелательные явления, что сказывается на КЖ пациентов и их комплаенсе. Уменьшение таких нежелательных явлений, как диарея, симптомы кишечной диспепсии (вздутие живота, урчание в животе, флатуленция) и повышение приверженности пациентов антибактериальной терапии возможно при назначении адъювантной терапии. Нивелированию указанных нежелательных явлений способствует прием препарата Закофальк NMX. Дополнительно у принимающих указанный препарат отмечено улучшение КЖ, особенно показателей RP и MH, что косвенно способствует повышению эффективности терапии и улучшению комплаентности пациентов.

Препарат Закофальк NMX целесообразно назначать при ЭТ Нр с 1-го дня лечения на весь период приема АБП (по 1 таблетке 2 раза в день либо 2 таблетки однократно).

\section{Литература}

1. Malfertheiner P., Megraud F., O'Morain C. et al. Management of Helicobacter pylori infection — the Maastricht V/Florence Consensus Report // Gut. - 2017; 66: 6-30.

2. Sugano K., Tack J., Kuipers E. et al. Kyoto global consensus report on Helicobacter pylori gastritis // Gut. - 2015; 64: 1353-67.

3. Маев И.В., Кучерявый Ю.А., Андреев Д.Н. Причины неэфффективности антигеликобактерной терапии // РЖГГК. - 2013; 6: 62-72.

4. Megraud F., Coenen S., Versporten A. et al. Helicobacter pylori resistance to antibiotics in Europe and its relationship to antibiotic consumption // Gut. - 2013; 62: 34-42.

5. Thung I., Aramin H., Vavinskaya V. et al. Review article: the global emergence of Helicobacter pylori antibiotic resistance // Aliment Pharmacol. Ther. - 2016; 43: 514-33.

6. Ивашкин В.Т., Маев И.В., Лапина Т.Л. и др. Рекомендации Российской гастроэнтерологической ассоциации по диагностике и лечению инсекции Helicobacter pylori у взрослых // Рос. журн. гастроэнт. гепатол. колопроктол. 2012; 22 (1): 87-9.

7. Кадыров Р.В., Асриян О.Б., Ковальчук С.А. Опросник «Уровень комплаентности». Монограсиия / Владивосток: Мор. гос. ун-т, 2014; 74 с.

8. Jung J., Cho I., Lee C. et al. Clinical Outcomes of Standard Triple Therapy Plus Probiotics or Concomitant Therapy for Helicobacter pylori Infection // Gut Liver. - 2017; doi: 10.5009/gnl17177. [Epub].

9. Song Z., Zhou L. Hybrid, sequential and concomitant therapies for Helicobacter pylori eradication: A systematic review and meta-analysis // World J. Gastroenterol. - 2016; 22 (19): 4766-75.

10. Vijay C. Antharam et al. Intestinal Dysbiosis and Depletion of Butyrogenic Bacteria in Clostridium difficile Infection and Nosocomial Diarrhea // J. Clin. Microbiol. - 2013; 51 (9): 2884-92.

11. Lin $\mathrm{Li}$ et al. The Effect of Helicobacter pylori Eradication on the Gastrointestinal Microbiota in Patients with Duodenal Ulcer // J. Gastrointestin. Liver Dis. - 2016; 25 (2): 139-46.

12. Izumi Shimbo et al. Effect of Clostridium butyricum on fecal flora in Helicobacter pylori eradication therapy // World J. Gastroenterol. - 2005; 11 (47): 7520-4.

13. Hamer H. et al. Review article: the role of butyrate on colonic function // Aliment. Pharmacol. Ther. - 2008; 27: 104-19.

14. Захаренко С.М. Повышение эфффективности антибиотикотерапии / М.: Прима Принт, 2018; 130 c.

15. Al-Eidan F., McElnay J., Scott M. et al. Management of Helicobacter pylori eradication - the influence of structured counselling and follow-up // Br. J. Clin. Pharmacol. - 2002; 53: 163-71.

16. Graham D., Lew G., Malaty H. et al. Factors influencing the eradication of Helicobacter pylori with triple therapy // Gastroenterology. - 1992; 102 (2): 493-6. 
17. Peura D., Crowe S. Helicobacter pylori // Sleisenger \& Fordtran's gastrointestinal and liver disease. Eds. M. Feldman, L. Friedman, L. Brandt. $9^{\text {th }}$ ed. / Philadelphia, Pa: Saunders Elsevier, 2010; chap. 50.

18. Peura D. Treatment of Helicobacter pylori infection // Therapy of digestive disorders. Ed. M.M. Wolfe / Philadelphia: Elsevier, 2006; p. 277.

19. Дурлештер В.М., Корочанская Н.В., Попандопуло К.И. и др. Современные подходы к хирургическому и медикаментозному лечению язв желудка. Учебно-методическое пособие / Краснодар, 2017.

\section{INCREASING COMPLIANCE IN THE ERADICATION THERAPY OF CHRONIC HP-ASSOCIATED GASTRITIS}

Professor E. Avalueva, MD; E. Skazyvaeva, Candidate of Medical Sciences; Professor I. Bakulin, MD; S. Sitkin, Candidate of Medical Sciences I.I. Mechnikov North-Western State Medical University, Saint Petersburg

Additional use of a butyric acid preparation (Zacofalk NMX) in the management of Helicobacter pylori in patients with chronic gastritis assists in reducing diarrheal syndrome and in improving quality of life, which increases patient compliance. Key words: gastroenterology, Helicobacter pylori, eradication therapy, chronic gastritis, compliance.

For citation: Avalueva E., Skazyvaeva E., Bakulin I. et al. Increasing compliance in the eradication therapy of chronic Hp-associated gastritis // Vrach. - 2018; 29 (12): 9-14. https://doi.org/10.29296/25877305-2018-12-02 